\title{
Atherectomy Cutter Length
}

National Cancer Institute

\section{Source}

National Cancer Institute. Atherectomy Cutter Length. NCI Thesaurus. Code C150230.

The length of the cutter component for atherectomy devices that are used to cut plaque/thrombus. 